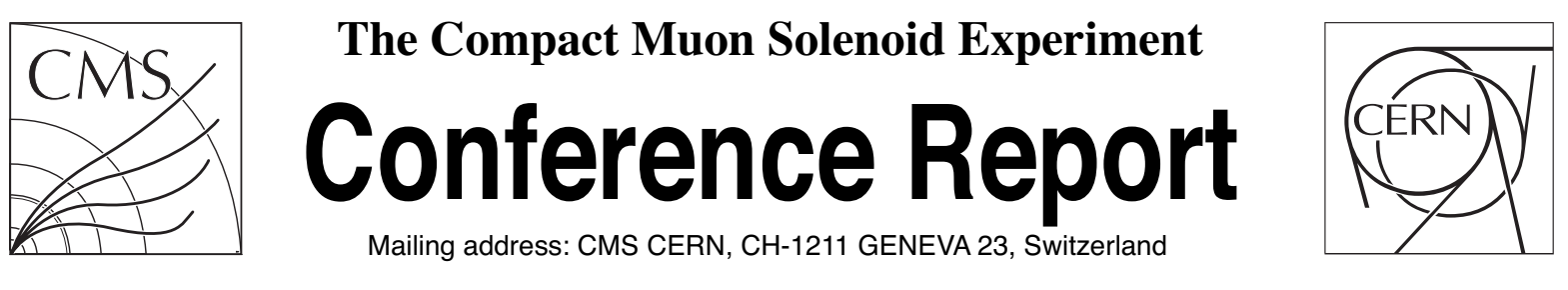

24 November 2018 (v3, 01 January 2019)

\title{
Long-term aging studies on Resistive Plate Chambers (RPC) of the CMS muon system for HL-LHC
}

Andrea Gelmi for the CMS Collaboration

\begin{abstract}
During the phase II of the LHC physics program, called High Luminosity LHC (HL-LHC), the accelerator will increase the instantaneous luminosity up to $5 \times 10^{34} \mathrm{~cm}^{-2} \mathrm{~s}^{-1}$, providing to experiments an additional integrated luminosity of about $3000 \mathrm{fb}^{-1}$ over 10 years of operation, starting in 2026. The present Compact Muon Solenoid (CMS) Resistive Plate Chambers (RPC) system has been certified for 10 LHC years operation, at maximum background rate of $300 \mathrm{~Hz} / \mathrm{cm}^{2}$ and $50 \mathrm{mC} / \mathrm{cm}^{2}$ as integrated charge. Based on Run2 data and assuming a liner dependence of the background rates as a function of the instantaneous luminosity, the expected rates and integrated charge at HL-LHC are about 600 $\mathrm{Hz} / \mathrm{cm}^{2}$ and $840 \mathrm{mC} / \mathrm{cm}^{2}$, respectively (including a safety factor of three). HL-LHC will be threfore a challenge for the RPC system since the expected operating conditions are much higher with respect those for which the detectors have been designed, and could induce non-recoverable aging effects which can alter the detector properties. A new longevity test is then needed to estimate the impact of HL-LHC conditions up to an integrated charge equivalent to the integrated luminosity of $3000 \mathrm{fb}^{-1}$, to confirm that the RPC system will survive to the harsher background conditions expected at HL-LHC. A dedicated consolidation program is ongoing at CERN Gamma Irradiation Facility (GIF++), where few RPC detectors are exposed to intense gamma radiation. The main detectors parameters (currents, rate, resistivity) are under monitoring as a function of the integrated charge and the performance studied with muon beam. After having collected a significant amount of the total irradiation preliminary results will be presented.
\end{abstract}




\title{
Long-term aging studies on Resistive Plate Chambers (RPC) of the CMS muon system for HL-LHC
}

\author{
Andrea Gelmi, on behalf of the CMS Collaboration \\ INFN and University of Bari, \\ Via Giovanni Amendola 173, IT-70125 Bari, Italy \\ andrea.gelmi@cern.ch
}

\begin{abstract}
During the phase II of the LHC physics program, called High Luminosity LHC (HL-LHC), the accelerator will increase the instantaneous luminosity up to $5 \times 10^{34} \mathrm{~cm}^{-2} \mathrm{~s}^{-1}$, providing to experiments an additional integrated luminosity of about $3000 \mathrm{fb}^{-1}$ over 10 years of operation, starting in 2026 . The present Compact Muon Solenoid (CMS) Resistive Plate Chambers (RPC) system has been certified for 10 LHC years operation, at maximum background rate of $300 \mathrm{~Hz} / \mathrm{cm}^{2}$ and 50 $\mathrm{mC} / \mathrm{cm}^{2}$ as integrated charge. Based on Run2 data and assuming a liner dependence of the background rates as a function of the instantaneous luminosity, the expected rates and integrated charge at HL-LHC are about $600 \mathrm{~Hz} / \mathrm{cm}^{2}$ and $840 \mathrm{mC} / \mathrm{cm}^{2}$, respectively (including a safety factor of three). HL-LHC will be threfore a challenge for the RPC system since the expected operating conditions are much higher with respect those for which the detectors have been designed, and could induce nonrecoverable aging effects which can alter the detector properties. A new longevity test is then needed to estimate the impact of HL-LHC conditions up to an integrated charge equivalent to the integrated luminosity of $3000 \mathrm{fb}^{-1}$, to confirm that the RPC system will survive to the harsher background conditions expected at HL-LHC. A dedicated consolidation program is ongoing at CERN Gamma Irradiation Facility (GIF++), where few RPC detectors are exposed to intense gamma radiation. The main detectors parameters (currents, rate, resistivity) are under monitoring as a function of the integrated charge and the performance studied with muon beam. After having collected a significant amount of the total irradiation preliminary results will be presented.
\end{abstract}

\section{Resistive Plate Chambers at CMS And FUture} PLANS

The muon detector system of the Compact Muon Solenoid (CMS) experiment [1] at the CERN Large Hadron Collider (LHC) consists of three types of detectors: Drift Tubes (DT) in the barrel region, Cathode Strip Chambers (CSC) in the endcap, and Resistive Plate Chambers (RPC) in both regions covering a pseudo rapidity region up to $|\eta|=1.9$ with 1056 chambers.

A CMS RPC chamber [2] consists of a double gas gap operated in avalanche mode to ensure reliable operation at high rates. Each gap consists of two $2 \mathrm{~mm}$ wide gas gap between two high resistive High-Pressure-Laminate (HPL) electrodes with $2 \mathrm{~mm}$ thickness. A humidified non-flammable threecomponent gas mixture of $95.2 \%$ freon $\left(\mathrm{C}_{2} \mathrm{H}_{2} \mathrm{~F}_{4}\right), 4.5 \%$ isobutane $\left(i-C_{4} H_{10}\right)$, and $0.3 \%$ sulphur hexafluoride $\left(S F_{6}\right)$ is used with a relative humidity of $\approx 40 \%$. The readout plane is located between the two gaps and consists of copper strips aligned in the $\eta$ direction with a pitch between 2.28 and 4.10 $\mathrm{cm}$ in the barrel and between 1.74 and $3.63 \mathrm{~cm}$ in the endcaps. The strip signals are asynchronously sent to the Front End Boards (FEBs) [3] which shape the signal before sending it to the RPC linkboard system and the CMS data acquisition system (DAQ).

The RPC system was designed to provide muon identification, excellent triggering, timing and momentum measurements at the LHC at the nominal luminosity of $1 \times 10^{34} \mathrm{~cm}^{-2} \mathrm{~s}^{-1}$. During the LHC Run1 and Run2 data taking the performance of the muon systems was outstanding [4]. In the second phase of the LHC physics program, called High Luminosity LHC (HL-LHC), the instantaneous luminosity will reach $5 \times 10^{34} \mathrm{~cm}^{-2} \mathrm{~s}^{-1}$, providing to CMS an additional integrated luminosity of about $3000 \mathrm{fb}^{-1}$ over 10 years of operation, starting in 2026.

\section{RPC LONGEVITY STUDIES}

When exposed to a prolonged radiation, gaseous detectors can suffer aging effects that typically results in: loss of efficiency, rise in spurious signal rates, increase in dark currents, etc. The main causes behind the detector performance deterioration are chemical processes largely occurring in the hot plasma inside electron multiplication region. Gaseous molecular fragments produced inside avalanches may form polymers growing on the electrodes [5]. The severity of aging increases with the radiation exposure and depends on many factors, such as the materials used for the electrodes, operational gas gain, gas mixture, impurities in the gas itself, gas flow rate, radiation rate, etc.

The present RPC system has been certified for 10 LHC years operation, at a maximum background rate of $300 \mathrm{~Hz} / \mathrm{cm}^{2}$ and a total integrated charge of $50 \mathrm{mC} / \mathrm{cm}^{2}$ [1]. Based on Run2 data and assuming a linear dependence of the background rates as a function of the instantaneous luminosity, the expected background rates and integrated charge will be about $600 \mathrm{~Hz} / \mathrm{cm}^{2}$ and $840 \mathrm{mC} / \mathrm{cm}^{2}$, respectively (including a safety factor of three) [6]. HL-LHC will therefore be a challenge for the RPC system since the new operating conditions are much higher with respect to those for which the detectors had been designed, and could induce non-recoverable aging 
effects that can alter the detector properties and performance. A new longevity test is then needed to estimate the impact of the HL-LHC conditions up to an integrated charge equivalent to the integrated luminosity of $3000 \mathrm{fb}^{-1}$, to confirm that the RPC system will survive to the expected HL-LHC conditions. Longevity studies will identify possible aging effects by monitoring the main detector parameters and performance as a function of the integrated charge.

\section{SET-UP AND TEST PROCEDURE}

A dedicated longevity study was set up at the CERN Gamma Irradiation Facility (GIF++) where it is possible to test full size detectors. The facility is equipped with a $13 \mathrm{TBq}$ Cs137 gamma source and a system of movable filters allows to varying the gamma flux, providing a fairly realistic simulation of the HL-LHC background conditions. A $100 \mathrm{GeV}$ muon beam, providing an excellent probes for detector performance studies, complements the source [7].

Since the maximum background rate is expected in the endcap region, in July 2016 the irradiation test was started at GIF++ using four spare endcap chambers: two RE2/2 and two RE4/2. Two different types of chambers have been used for this test because the endcap RPC production has been performed in two periods: in 2005 for all RPCs in the endcap system, except the RE4/2 and RE4/3 chambers, which were made in 2012-2013. In order to study the longevity of the detectors, two of the four chambers (one RE2/2 and one RE4/2), are continuously operated under gamma irradiation, while the remaining two chambers are turned on only from time to time and are used as reference.

The main detector parameters are monitored and periodically compared with those of the reference chambers (currents and counting rates at several background conditions, noise and dark current, etc). Moreover, when the muon beam at GIF++ is available, the detector performance is studied at different irradiation fluxes. The method for the data analysis is described in Ref. [8].

All measurements are performed under controlled environmental and gas conditions.

At present, about $478 \mathrm{mC} / \mathrm{cm}^{2}$ and $251 \mathrm{mC} / \mathrm{cm}^{2}$ have been integrated for RE2/2 and RE4/2 irradiated detectors, which corresponds respectively to approximately $57 \%$ and $30 \%$ of the expected HL-LHC integrated charge. The integrated charge versus time is shown in Fig.1.

\section{DETECTOR PARAMETERS MONITORING}

The rate and current are measured all the time for both irradiated and non-irradiated reference chambers. In order to spot possible degradations of the electrodes' surface due to the irradiation, the detector noise rate and dark current are periodically measured. Figure 2 shows the currents (top) and noise rate (bottom) at the working point as a functions of the integrated charge for the RE2/2 irradiated and reference chambers. No significant variations have been observed so far.

The current and rate with background radiation are periodically measured as well. To exclude the dependence on

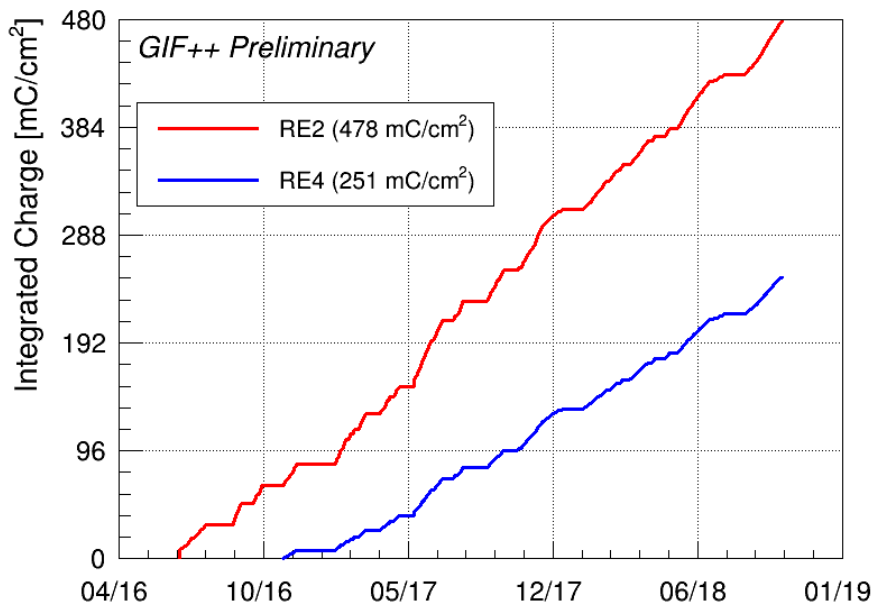

Fig. 1. Integrated charge versus time, accumulated during the longevity test at GIF++ for RE2/2 (red) and RE4/2 (blue) irradiated chambers. The RE4/2 chamber has been turned on a few months later because of total gas flow limitations. Different slopes account for different irradiation conditions during data taking.

the external parameters, the ratios of the irradiated and the reference chambers is measured as a function of the integrated charge. Figure 3 top shows the RE2/2 current and rate ratio.

The measurements show a decreasing trend at the beginning of the irradiation period, up to $\approx 300 \mathrm{mC} / \mathrm{cm}^{2}$, when the operating conditions, in terms of gas flow rate and relative gas humidity, were too low with respect to the high gamma background rate. These operating conditions leads to the electrodes' resistivity increase, which caused the observed rate and current decrease.

The electrodes' resistivity increase is confirmed by the measurements performed running the RPCs filled with pure Argon gas. Figure 3 bottom shows the coherent correlation between the RE2/2 currents ratio (red) and the resistivity variation (blue). The resistivity variation allows to cancel out the dependence on the environmental conditions, and is defined as:

$$
\rho_{v a r}=\frac{\rho_{i r r}-\rho_{r e f}}{\rho_{i r r}}
$$

These plots show also that the resistivity increase is a recoverable effect, in fact the resistivity start to decrease, and the current and rate to increase, when the gas flow and the gas relative humidity have been increased.

\section{DETECTOR PERFORMANCE MONITORING}

The detector performance has been tested before starting the longevity test and repeated after different irradiation periods corresponding to $18 \%\left(153 \mathrm{mC} / \mathrm{cm}^{2}\right), 31 \%\left(257 \mathrm{mC} / \mathrm{cm}^{2}\right)$, $45 \%\left(378 \mathrm{mC} / \mathrm{cm}^{2}\right)$ and $51 \%\left(431 \mathrm{mC} / \mathrm{cm}^{2}\right)$, of the total integrated charge expected by the end of HL-LHC. Figure 4 shows the RE2/2 irradiated chamber efficiency as a function of the effective High Voltage (voltage normalized at the standard 

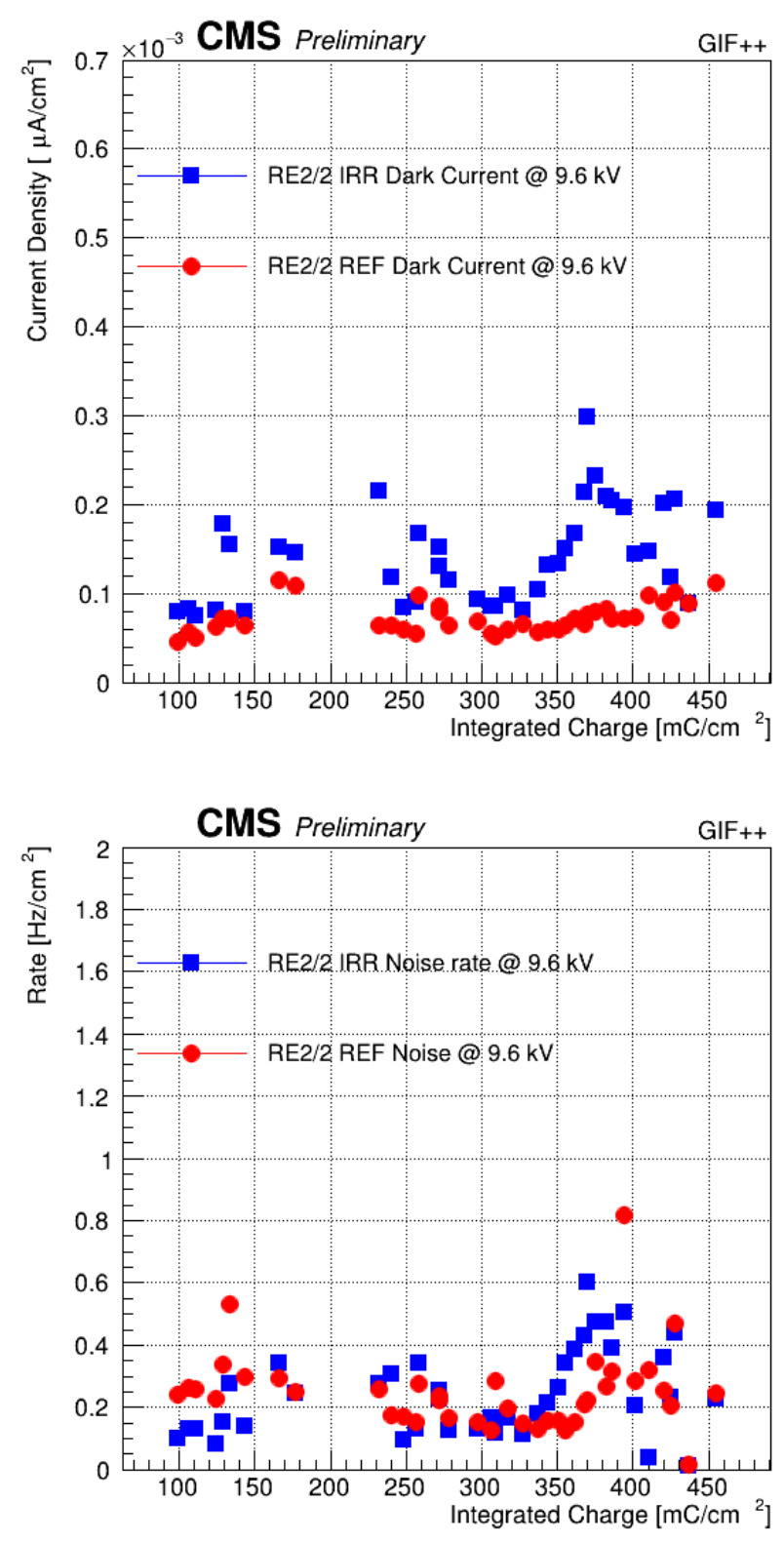

Fig. 2. Dark current (top) and noise rate (bottom) versus the integrated charge, for RE2/2 irradiated (blues) and reference (red) chamber, at the working point voltage.

temperature and pressure), without irradiation (top), and with a gamma background rate of $\approx 600 \mathrm{~Hz} / \mathrm{cm}^{2}$ (bottom).

The performance without background is stable in time and we do not observe any efficiency degradation or working point shift. With a background rate of $\approx 600 \mathrm{~Hz} / \mathrm{cm}^{2}$ the efficiency remains stable at working point, but we observe an $100 \mathrm{~V}$ shift, starting from $45 \%$ of the expected integrated charge.

The working point shift is related to the electrodes' resistivity increase, since the effective voltage applied to the electrodes (HV) is reduced by the voltage drop across the electrodes, which is proportional to the current (I) produced by the ionizing particles and to the bakelite resistance (R)
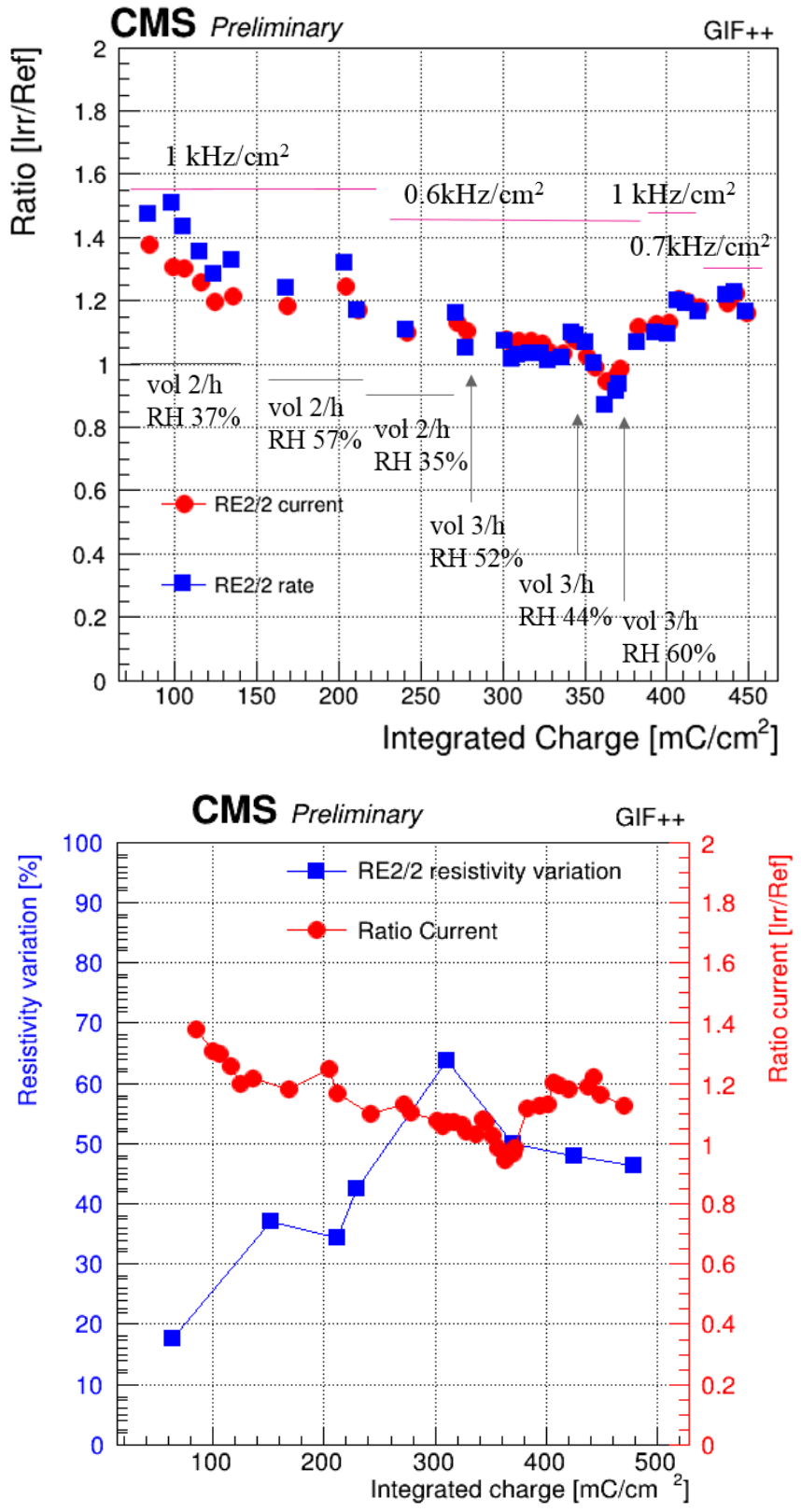

Fig. 3. Top: RE2/2 current (red) and rate (blues) ratio between irradiated and reference chamber as a function of the integrated charge. Bottom: RE2/2 current ratio (red) and resistivity variation (blue).

[9], [10]. The effective voltage applied to the gas $\left(H V_{\text {gas }}\right)$ is therefore defined as:

$$
H V_{\text {gas }}=H V-R I
$$

The detector operation regime is invariant with respect to $H V_{\text {gas }}$, therefore the efficiency as a function of $H V_{\text {gas }}$ does not depends anymore on the background radiation and on the bakelite resistance. Figure 5 represent the RE2/2 irradiated chamber efficiency curves at different background radiation (up to $\approx 600 \mathrm{~Hz} / \mathrm{cm}^{2}$ ) and at different integrated charge. All the efficiency curves overlap and we do not observe anymore 

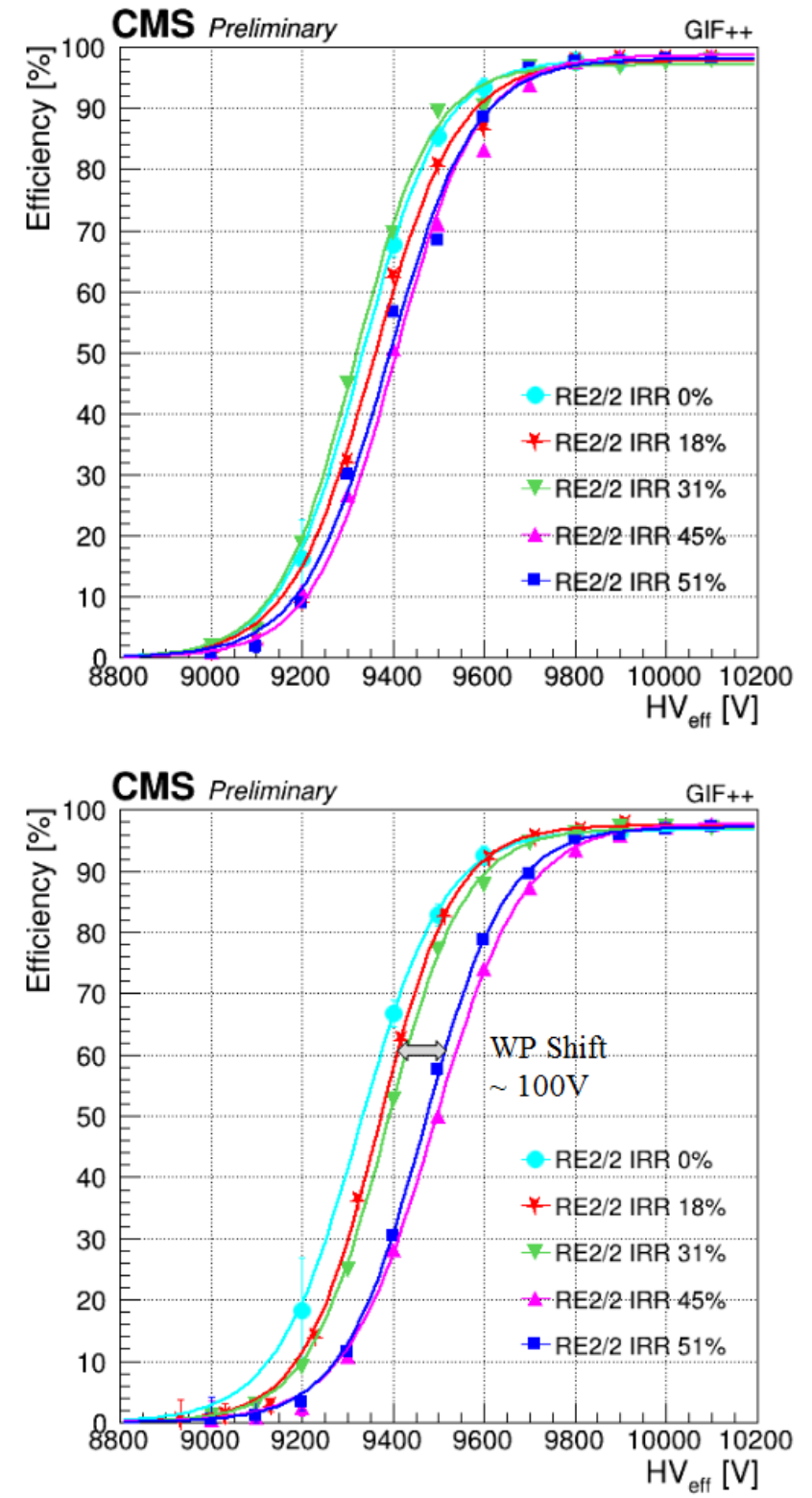

Fig. 4. RE2/2 irradiated chamber efficiency as a function of the effective $\mathrm{HV}$, taken with no irradiation (top) and under a gamma background rate of about $600 \mathrm{~Hz} / \mathrm{cm}^{2}$ (bottom). The efficiency is measured during different Test Beams (TB) corresponding to different fractions of the target charge to integrate.

the working point shift, since the electrodes' resistivity increase effect has been removed.

The RE2/2 irradiated chamber efficiency at the working point as a function of the background rate is shown in Fig. 5. The efficiency is stable in time, with a $2 \%$ decrease at the highest expected background rate, $600 \mathrm{~Hz} / \mathrm{cm}^{2}$. This also proves that the detectors are able to work efficiently at the maximum expected rate during HL-LHC.

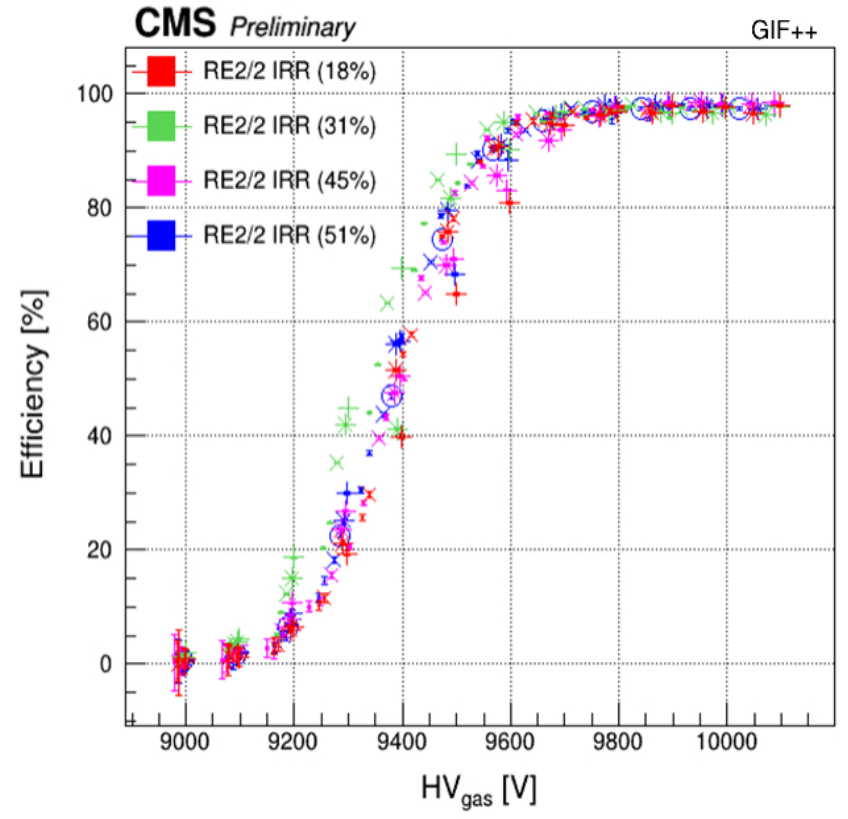

Fig. 5. RE2/2 irradiated chamber efficiency as a function of the HV gas, at different background irradiations and at different integrated charge values.

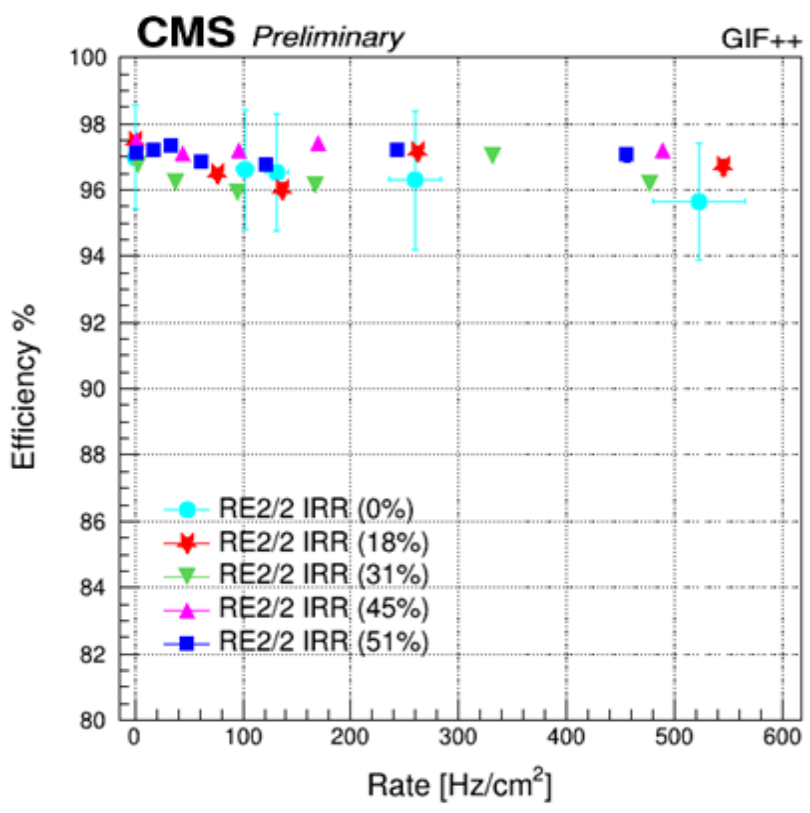

Fig. 6. RE2/2 irradiated chamber efficiency at working point as a function of the background rate at different integrated charge values.

\section{CONCLUSions}

In view of HL-LHC the CMS RPC detectors must prove that they are able to work efficiently at the expected operating conditions for the entire period. Longevity studies on spare RPCs are ongoing at GIF++ under controlled conditions. From preliminary results no evidence of any aging effect has been observed so far. The main detectors parameters are stable, 
unless minor variations such as the electrodes' resistivity, that did not affected the performance which remains stable up to $57 \%$ of the expected integrated charge. Further investigations are needed to get closer to the final integrated charge of $840 \mathrm{mC} / \mathrm{cm}^{2}$.

\section{REFERENCES}

[1] CMS Collaboration, The CMS muon project: Technical Design Report, CERN, Geneva Switzerland, LHC Experiments Committee (1997) [CMS-TDR-3]

[2] G. Pugliese [CMS Muon Collaboration], The RPC system for the CMS experiment, 2006 IEEE NSS Conference Record N24-3, January 2007.

[3] F. Loddo et al., Front-end for the RPC detector in CMS, in Proceedings of the 4th International Workshop on Resistive Plate Chamber and Related Detectors, Napoli, Italy, 15-16 October 1997.

[4] A. M. Sirunyan et al. [CMS Collaboration], Performance of the CMS muon detector and muon reconstruction with proton-proton collisions at $\sqrt{s}=13 \mathrm{TeV}$, [arXiv:1804.04528 [physics.ins-det]].

[5] M. Abbrescia et al., HF production in CMS-Resistive Plate Chambers, Nucl. Phys. B 158 (2006) 30-34

[6] CMS Collaboration, The Phase-2 Upgrade of the CMS Muon Detectors, CERN, Geneva Switzerland, LHC Experiments Committee (2017) [CMS-TDR-016]

[7] R. Guida [EN and EP and AIDA GIF++ Collaborations], GIF++: A new CERN Irradiation Facility to test large-area detectors for the HL-LHC program, PoS ICHEP 2016 (2016) 260.

[8] M. Abbrescia et al., Cosmic ray tests of double-gap resistive plate chambers for the CMS experiment, Nucl. Instrum. Meth. A 550 (2005) 116. doi:10.1016/j.nima.2005.06.074

[9] G. Pugliese et al., Aging study for resistive plate chambers of the CMS muon trigger detector, Nuclear Instruments and Methods in Physics Research A 515, 342347 (2003).

[10] G. Aielli et al., Further advances in aging studies for RPCs, Nuclear Instruments and Methods in Physics Research A 515, 335 (2003). 